\title{
A High Performance Liquid Chromatography-Mass Spectrometry (HPLC-MS) Qualitative Detection Method Developed for In Vivo Analyses of Toxin Orellanine from the Cortinarius orellanus Fr.-Part II
}

\author{
Ilia Brondz ${ }^{1}$ and Anton Brond $z^{2}$ \\ ${ }^{1}$ Department of Biology, University of Oslo, Blindern, P. O. Box 1066, 0316 Oslo, Norway \\ ${ }^{2}$ Department of Chemistry, Norwegian University of Science and Technology, 7491 Trondheim, Norway
}

Correspondence should be addressed to Ilia Brondz, ilia.brondz@bio.uio.no

Received 26 December 2011; Accepted 15 January 2012

Academic Editors: L. Chen, R. Nageswara Rao, T. Richard, and S. Valsecchi

Copyright (C) 2012 I. Brondz and A. Brondz. This is an open access article distributed under the Creative Commons Attribution License, which permits unrestricted use, distribution, and reproduction in any medium, provided the original work is properly cited.

\begin{abstract}
The high-performance liquid chromatography—mass spectrometry (HPLC-MS) qualitative analysis method in negative mode of detection was used to record the presence of orellanine in the stomach contents of rats after ingestion of Cortinarius orellanus. Intoxication with orellanine causes acute renal failure (ARF), which is characterized by sudden loss of the ability of the kidneys to excrete wastes. The detection method presented here can be used as a platform for future development of analytical procedures for detecting orellanine in cases of human intoxication by toadstools. The presence of orellanine in animal stomach contents was analytically recorded and compared with standard orellanine, which was donated by the R\&D Department of Jupiter Ltd, Norway. The MS fragmentation described in the literature and the MS fragmentation of standard samples of isolated orellanine verified the presence of orellanine in the stomach contents of laboratory animals. The presence of a diglucoside of orellanine, which was described as part of the toxic orellanine complex by Spiteller et al., in the stomach contents of animals, was also verified using HPLC-MS.
\end{abstract}

\section{Introduction}

In the last few decades, edible mushrooms have represented a growing segment in the diets of western European and north American populations, and these delicate-tasting and protein-rich vegetables have been grown on an industrial scale; however, forest mushrooms have also experienced increasing popularity at the dining table. A significant increase in the production and import of forest mushrooms from eastern Europe and Poland has been observed, but self-collection of forest mushrooms has also become more popular in recent decades. The possibility of mistaking nonedible for edible mushrooms is significant among amateur collectors.

Epidemic outbreaks of mass intoxication by pseudoedible mushrooms were observed in Poland in 1952-1957. Later in 1957, the cause of these outbreaks was described by Grzymala as intoxication by a member of the Cortinarius family [1]. The toxin orellanine from Cortinarius orellanus was first isolated by Grzymala in 1962 as part of a mixture with other substances. The chemical structure of orellanine was defined as $3,3^{\prime}, 4,4^{\prime}$-tetrahydroxy-2,2' -bipyridine- $N-N^{\prime}$-dioxide [2]. Several studies have been performed over the years to support the initial proposition of its chemical structure. The study performed by Holmdahl et al. supported the correctness of this chemical structure based on ${ }^{1} \mathrm{H}-\mathrm{NMR}$ and ${ }^{13} \mathrm{C}-\mathrm{NMR}$ spectra [3]. However, a doubt was expressed regarding the level of the toxic effects of orellanine, a toxin from the mushroom $C$. orellanus, compared with other bipyridines [4]. The exact mechanism underlying the biotoxicity of orellanine remains under discussion; Richard 
et al. in [4] compared the toxicity of bipyridines with the toxicity of orellanine.

The structures of bipyridines $\left(2,2^{\prime}\right.$-bipyridine and $4,4^{\prime}$ bipyridine) resemble that of orellanine; however, not all bipyridines exhibit high toxicity. The two substances paraquat in the salt form $\left(N, N^{\prime}\right.$-dimethyl-4,4' -bipyridinium dichloride) and diquat in the salt form $\left(1,1^{\prime}\right.$-ethylene- $2,2^{\prime}$ bipyridyldiylium dibromide) have been used as pesticides. The drug inamrinone is a 5 -amino-3, $4^{\prime}$-bipyridine- $6(1 H)$ one that is used to treat patients with congestive heart failure [5], and milrinone (2-methyl-6-oxo-1,6-dihydro-3,4'bipyridine-5-carbonitrile) is a phosphodiesterase 3 inhibitor that potentiates the effect of cyclic adenosine monophosphate (cAMP).

Paraquat has the ability to interrupt electron transfer processes in biological systems, and its toxicity arises from eruption of electron transport in living organisms. Diquat may be fatally toxic to humans [6]; its ingestion may cause nausea, vomiting, diarrhea, gastrointestinal discomfort, chest pain, liver damage, and failure of kidney function. In fatal cases, the death course is of 2-14 days' duration [7-10]. Diquat toxicity somewhat resembles the toxicity of orellanine, by injuring the kidneys and exhibiting a prolonged intoxication period.

The toxins of C. orellanus have a prolonged latent period after ingestion, which, together with the thermo- and photolability of orellanine, have hampered the development of an analytical method for the direct analytical diagnosis of $C$. orellanus poisoning using body fluids [11, 12]. In addition to orellanine, the C. orellanus toxins include cortinarin A, B, and $\mathrm{C}$, which are also nephrotoxins, the nontoxic orellinine, and the orellanine- $4,4^{\prime}$-diglucopyranoside proposed in [13]. The toxicity of the diglucoside of orellanine remains unclear. Its structural formula is shown in Figure 1. Because orellanine is thermolabile and orellanine- $4,4^{\prime}$-diglucopyranoside is impossible to analyze by GC or GC-MS without derivatization, HPLC-MS was chosen as an analytical instrumental method, especially as we hoped to detect orellanine- $4,4^{\prime}$ diglucopyranoside in stomach contents after ingestion of a meal containing C. orellanus.

The aim of this study was to develop a method for orellanine detection in stomach juice after ingestion of a meal containing $C$. orellanus and to study the degradation of orellanine- $4,4^{\prime}$-diglucopyranoside. The present study is a further development of [11], which was presented at the Separation Science Europe 2011 Conference and Exhibition (October 10-11, 2011) at the Royal Institution in London, UK.

\section{Materials and Methods}

2.1. Instrumentation and Conditions. The instruments used were an HPLC chromatographic system comprising an HP 1100 instrument (Hewlett-Packard, Palo Alto, CA, USA) equipped with an Ascentis Express C18 $2 \mu \mathrm{m}$ column measuring $50.0 \mathrm{~mm} \times 2.1 \mathrm{~mm}$ I.D. (Supelco, Bellefonte, PA, USA). Chromatographic conditions were as follows. The composition of the mobile phase was acetonitrile p.a. quality (Merck, Darmstadt, Germany) and $0.1 \mathrm{~N}$ formic acid (Merck) in deionized water $(60: 40, \mathrm{v}: \mathrm{v})$. The extracts from the stomach contents of 13 animals were individually for each animal eluted isocratically using a flow rate of $0.25 \mathrm{~mL} / \mathrm{min}$. The injection volume was $20 \mu \mathrm{L}$. The HPLC system was coupled online to a Quattro MS-MS triple-quadruple mass spectrometer (Micromass, Altrincham, UK) equipped with a pneumatically assisted electrospray ionization source. The effluent entered the mass spectrometer through an electrospray capillary set at $3.0 \mathrm{kV}$ at a source block temperature of $90^{\circ} \mathrm{C}$ and a desolvation gas temperature of $150^{\circ} \mathrm{C}$. Nitrogen was used at 610 and $80 \mathrm{~L} / \mathrm{h}$, respectively. The ion source parameters were optimized regarding the negative molecular ions. Mass spectra between $\mathrm{m} / \mathrm{z} 120$ and $\mathrm{m} / \mathrm{z} 600$ were obtained at a scan speed of $200 \mathrm{~m} / z$ unit scan/s with a mass resolution corresponding to 1 unit at half-peak height. Data acquisition and processing were performed using a MassLynx 4.0 SP4 data system (Waters). The mass spectra were recorded using negative detection; the mass spectrum is shown in Figure 2.

2.2. Standard and Biological Samples. The orellanine standard and biological samples were donated by Jupiter AS, Norway. C. orellanus mushrooms were collected in the vicinity of Oslo and freeze-dried to a constant weight before their use in experiments.

2.3. Sample Preparation. The biological samples of stomach contents and the procedure for recovered biological samples were as follows. Animals (Mus rattus L.) were fed pellets containing dried C. orellanus (1\%), glucose (4\%), hydrolyzed protein $(20 \%)$, fat $(10 \%)$, and ground cereals $(65 \%)$ and were given water in quantum satis. After $6 \mathrm{~h}$, the stomach contents of the animals were drained via a probe, without decapitation of the animals. The stomach contents obtained from 13 animals were kept in liquid nitrogen for each animal separately. Stomach content samples $(10 \mathrm{mg})$ from each animal were homogenized in an ultrasound bath at $0^{\circ} \mathrm{C}$ and centrifuged at $10,000 \mathrm{rpm}$ for $15 \mathrm{~min}$ at $4^{\circ} \mathrm{C}$, the supernatant was separated from the precipitate and lyophilized, and samples were kept in liquid nitrogen. Prior to analysis, the samples were adjusted to a volume of $0.5 \mathrm{~mL}$ using mobile phase.

\section{Results}

The mass spectrum presented in Figure 2 is in compliance with the spectra published in $[2,11,12,14]$ and exhibits base ion $[\mathrm{M}-\mathrm{H}]^{-}$at $251 \mathrm{~m} / \mathrm{z}$. The other characteristic fragment ions described previously $[2,11,12,14]$ were present in the spectrum. Overall, the spectrum was characteristic for orellanine and was identical to that of standard orellanine. The diglucoside of orellanine was not detected in stomach contents after $6 \mathrm{~h}$. The concentration of orellanine in stomach content was $0.2 \mathrm{mg} / \mathrm{mL}$. 


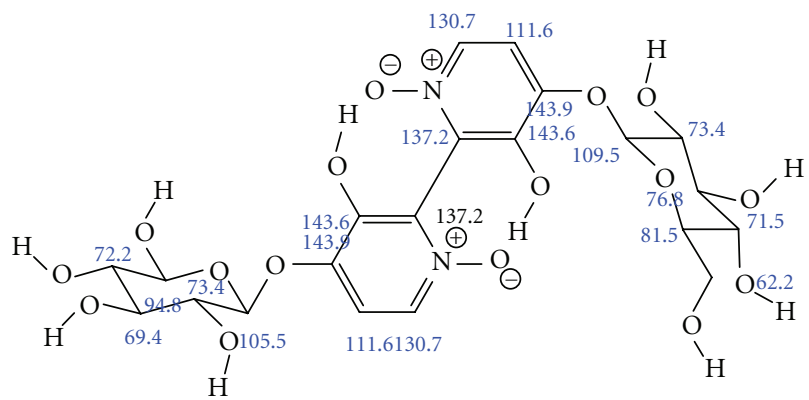

FIGURE 1: The structural formula of 3,3'-dihydroxy-4,4'-bis $(((2 S, 3 R, 4 S, 5 S, 6 R)-3,4,5$-trihydroxy-6-(hydroxymethyl)tetrahydro- $2 H$-pyran2yl)oxy)-[2,2' -bipyridine $] 1,1^{\prime}$-dioxide with chemical shifts in vicinity of every carbon atom. The chemical shifts are theoretically calculated for ${ }^{13} \mathrm{C}$ NMR by using ChemBioDraw Ultra 12.0 Suite. http://scistore.cambridgesoft.com/.

\section{Discussion}

Grzymala [1] described mass poisoning with C. orellanus. C. orellanus main toxin, orellanine, was proposed as the cause and was studied intensely by several authors [2-4], who worked out its structural formula. It was not difficult to demonstrate the stable appearance of orellanine in all studied samples as the base ions $[\mathrm{M}-\mathrm{H}]^{-}$at $251 \mathrm{~m} / \mathrm{z}$ and related fragment ions, which was the main aim of this study. The MS spectrum obtained was in good agreement with the results of previous publications.

Spiteller et al. [13] proposed the existence of the orellanine- $4,4^{\prime}$-diglucopyranoside molecule shown in Figure 1 in $C$. orellanus and in other toxic mushrooms of the Cortinarius family. It remains to be determined whether orellanine is a degradation product of orellanine- $4,4^{\prime}$ diglucopyranoside or an artifact of sample preparation. The formation of artifacts from substances present in mushrooms and their assumption as original and authentic substances are not unknown phenomena. For decades, the presence of infractine in $C$. infractus was accepted as a well-known fact, and infractine was also found in green plants, until Brondz et al. [15] discovered that infractine was an artifact that arose from a mistake during sample preparation.

The appearance of artifacts in this type of analysis is not surprising, as microbiologists, mycologists, or botanists are performing the chemical analytical work, rather than chemists. The possibility of recording the intact orellanine 4,4'-diglucopyranoside using gas chromatography-mass spectrometry with supersonic molecular beams (GC-MS with $\mathrm{SMB}$ ), which has been used to analyze orellanine in stomach contents [12], is negligible because of possible thermal degradation, even if the thermal degradation that results from this instrumental modification [12] is lower than that observed in conventional GC-MS. The HPLC-MS seems to be the tool of choice.

The long-standing dispute regarding the structure/ activity of orellanine [4] and the contradictory reports of orellanine solubility published by Spiteller et al. [13] on one side and Kürnsteiner and Moser [16] on the other could not be addressed in this study because of the absence of the characteristic ion that could represent orellanine- $4,4^{\prime}$ diglucopyranoside.

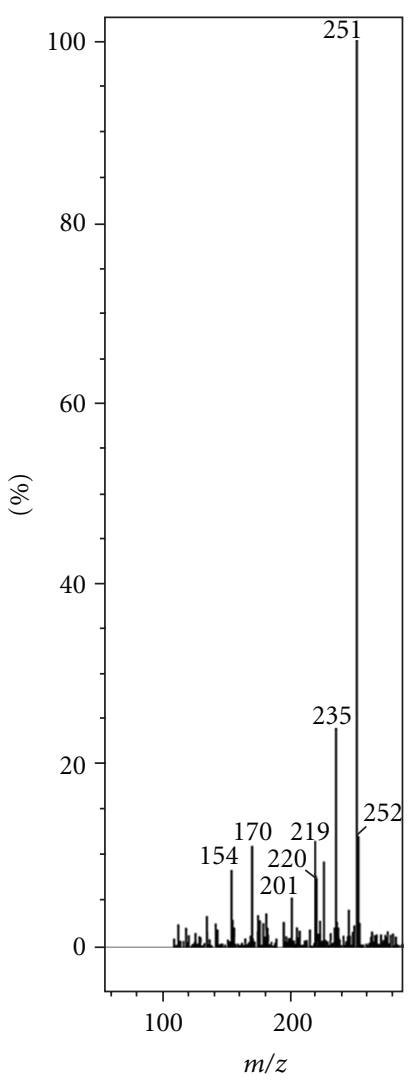

Figure 2: The MS spectrum of orellanine. The analytical conditions are described in the text.

The possible explanations for this negative result include the fact that mass spectrometry suffers from suppression of ion production, which is not surprising in the case of HPLC/MS, and the effects of contamination from ion souse because of the high boiling point of orellanine$4,4^{\prime}$-diglucopyranoside and other substances present in the analyzed sample. Another explanation could be the relatively long period of exposure of orellanine-4, $4^{\prime}$ diglucopyranoside to digestive juice in the stomach, which has an acidic $\mathrm{pH}$. Orellanine- $4,4^{\prime}$-diglucopyranoside could be hydrolyzed during the 6 h exposure to digestive juice in the 
stomach [13]. The modification of the analytical procedure may help recognize orellanine- $4,4^{\prime}$-diglucopyranoside if it is present in these mushrooms. This will cast light on the mechanism underlying the intoxication associated with these mushrooms, which at present is not sufficiently clear and remains disputable. Orellanine- $4,4^{\prime}$-diglucopyranoside does not seem to be a highly toxic substance and, as it is a water soluble, it should be excreted by the kidneys or in feces. However, there are examples of the hydrolysis of nontoxic glycosides to highly toxic substances: the aglycone part of the original molecule. An example of this is amygdalin, $\{[(6-O-\beta-\mathrm{D}$-glucopyranosyl $)-\beta-\mathrm{D}$ glucopyranosyl] oxy (phenyl)acetonitrile, which was isolated initially from the seeds of the bitter almond tree (Prunus dulcis). Amygdalin is hydrolyzed to mandelic acid, Dglucose, and ammonia in hydrochloric acid; however, in sulfuric acid, it is hydrolyzed to D-glucose, benzaldehyde, and the highly toxic cyanic acid [17], emulsin also hydrolyzes amygdalin it into benzaldehyde, cyanide, and two molecules of glucose.

In publication [13], several important data are missing regarding the exact identification of orellanine- $4,4^{\prime}$-diglucopyranoside.

(1) The absence of the correct nomenclature according to IUPAC rules. It seems that, as depicted in [13], the substance should be named 3,3'-dihydroxy$4,4^{\prime}$-bis $(((2 S, 3 R, 4 S, 5 S, 6 R)-3,4,5$-trihydroxy-6(hydroxymethyl)tetrahydro-2H-pyran-2yl)oxy)[2,2' -bipyridine $] 1,1^{\prime}$-dioxide.

(2) The exact mass of the molecule depicted in [13] should be 576.14 and its exact molecular weight 576.46. Neither the exact mass nor the exact molecular weight was given in [13]. The MS spectrum was not documented in [13] in the usual way.

(3) The ${ }^{13} \mathrm{C}$-NMR chemical shifts presented in [13] for some carbons are in agreement with theoretically calculated chemical shifts (Figure 1, the chemical shifts are shown in the vicinity of related carbon atoms in the structure of $3,3^{\prime}$-dihydroxy-4, $4^{\prime}$ bis $(((2 S, 3 R, 4 S, 5 S, 6 R)$-3,4,5-trihydroxy-6-(hydroxymethyl)tetrahydro-2H-pyran-2yl)oxy)-[2,2'-bipyridine] $1,1^{\prime}$-dioxide); however, in most cases, these chemical shifts deviated strongly from theoretical calculations.

(4) Following the description of the isolation of orellanine-4,4'-diglucopyranoside in [13], it should be relatively easy to obtain this substance, possibly even with good yield, for the study of its toxicological and chemical properties. However, we could not find information in the published literature regarding these aspects, despite [13] was published in 2003.

\section{Conclusion}

The study presented here is a further development of [11]. The study reported in [11] was based on results recorded from only one individual animal. In the present study, a group of 13 individual animals was used to obtain 13 individual stomach content extracts after animals were fed a meal containing $C$. orellanus. In all samples, the presence of orellanine was recorded by HPLC-MS. This study may represent the basis for the analysis of stomach contents in cases of human intoxication with mushrooms containing orellanine. Kidney biopsy followed by histological examination or histological examination of stomach contents is the diagnostic procedure used currently. However, the preparation of mushrooms for meals and the partial digestion of meals in the stomach at the time of appearance of the intoxication symptoms destroy most of the fine structure of the ingested material and prevent the establishment of unequivocal histological conclusions [12] regarding intoxication by orellanine-containing mushrooms. The direct detection of orellanine is needed for correct medication in these cases.

\section{Acknowledgments}

The authors are grateful to Jupiter Ltd, Norway, for donation of orellanine standard, biological samples, and financial support. The authors are grateful to Jon Reierstad from the Technical Department of the University of Oslo, Norway, for technical assistance in the preparation of figures.

\section{References}

[1] S. Grzymala, "Massenvergiftungen durch den orangefuchsigen Hautkopf," Zeitschrift für Pilzkunde, vol. 23, pp. 139-142, 1957.

[2] W. Z. Antkowiak, R. Antkowiak, E. Wyrzykiewicz, and G. Czerwiński, "Mass spectral fragmentation of orellanine and its tetramethyl ether with regard to their facile thermal and photochemical deoxygenation," Heterocycles, vol. 39, no. 2, pp. 477-484, 1994.

[3] J. Holmdahl, J. Ahlmén, S. Bergek, S. Lundberg, and S.-A. Persson, "Isolation and nephrotoxic studies of orellanine from the mushroom Cortinarius speciosissmus," Toxicon, vol. 25, no. 2, pp. 195-199, 1987.

[4] J.-M. Richard, G. Taillandier, and J. L. Benoit-Guyod, “A quantitative structure-activity relationship study on substituted pyridines as a contribution to the knowledge of the toxic effects of orellanine, a toxin from the mushroom Cortinarius orellanus," Toxicon, vol. 23, no. 5, pp. 815-824, 1985.

[5] N. A. Klein, S. J. Siskind, W. H. Frishman, E. H. Sonnelblick, and T. H. LeJemtel, "Hemodynamic comparison of intravenous amrinone and dobutamine in patients with chronic congestive heart failure," American Journal of Cardiology, vol. 48, no. 1, pp. 170-175, 1981.

[6] Chevron Chemical Company, "Diquat herbicide h/a for aquatic plant treatment. Agricultural Chemicals Division," Agricultural Chemicals Division. No. 8616 DIQ-45, Chevron Chemical Company, San Francisco, Calif, USA, 1986.

[7] Occupational Health Services, Material Safety Data Sheets, OHS, Secaucus, NJ, USA, 1986.

[8] WSSA Herbicide Handbook Committee, Herbicide Handbook of the Weed Science Society of America, WSSA, Champaign, Ill, USA, 6th edition, 1989.

[9] Occupational Health Services, MSDS for Diquat, OHS, Secaucus, NJ, USA, 1991. 
[10] W. J. Hayes and E. R. Laws, Eds., Handbook of Pesticide Toxicology, vol. 3 of Classes of Pesticides, Academic Press, New York, NY, USA, 1990.

[11] I. Brondz, E. Nevo, S. Wasser, K. Høiland, F. Størmer, and A. Brondz, "Method development for in vivo analyses of toxin orellanine from the toad mushroom Cortinarius orellanus," in Proceedings of the Separation Science Europe Conference, The Royal Institution, London, UK, October 2011.

[12] I. Brondz, E. Nevo, S. Wasser, and A. Brondz, "A direct Gas Chromatography-Mass Spectrometry (GC-MS) method for the detection of orellanine present in stomach content. Part I," Journal of Biophysical Chemistry, vol. 3, no. 1, 2012.

[13] P. Spiteller, M. Spiteller, and W. Steglich, "Occurrence of the fungal toxin orellanine as a diglucoside and investigation of its biosynthesis," Angewandte Chemie_-International Edition, vol. 42, no. 25, pp. 2864-2867, 2003.

[14] J.-M. Richard and J. Ulrich, "Mass spectrometry of orellanine, a mushroom toxin, and of related bipyridine- $N$-oxides," Biomedical and Environmental Mass Spectrometry, vol. 18, no. 1, pp. 1-4, 1989.

[15] I. Brondz, D. Ekeberg, K. Høiland, D. S. Bell, and A. R. Annino, "The real nature of the indole alkaloids in Cortinarius infractus: evaluation of artifact formation through solvent extraction method development," Journal of Chromatography $A$, vol. 1148, no. 1, pp. 1-7, 2007.

[16] H. Kürnsteiner and M. Moser, "Isolation of a lethal toxin from Cortinarius orellanus Fr," Mycopathologia, vol. 74, no. 2, pp. 65-72, 1981.

[17] J. W. Walker and V. K. Krieble, "CLIII.- - the hydrolysis of amygdalin by acids. Part I," Journal of the Chemical Society, vol. 95, pp. 1369-1377, 1909. 


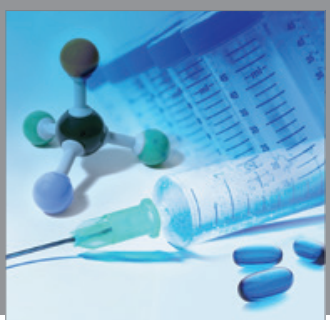

International Journal of

Medicinal Chemistry

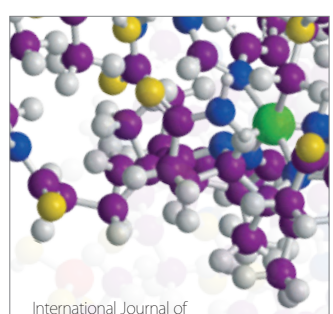

Carbohydrate Chemistry

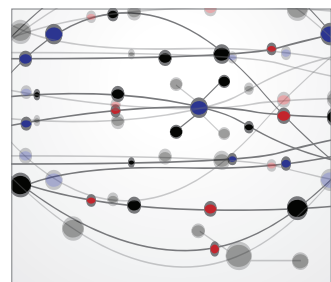

The Scientific World Journal
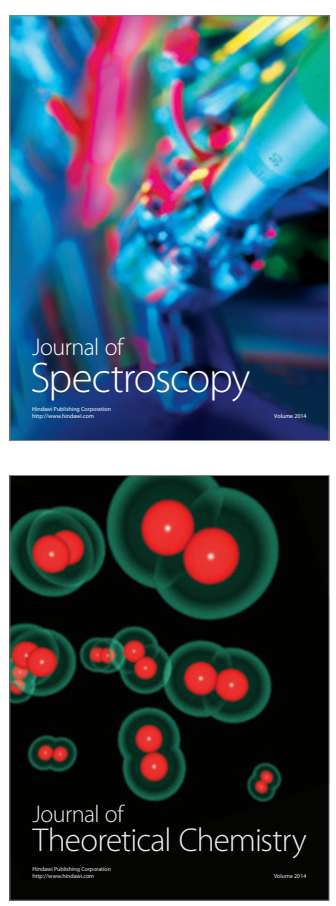
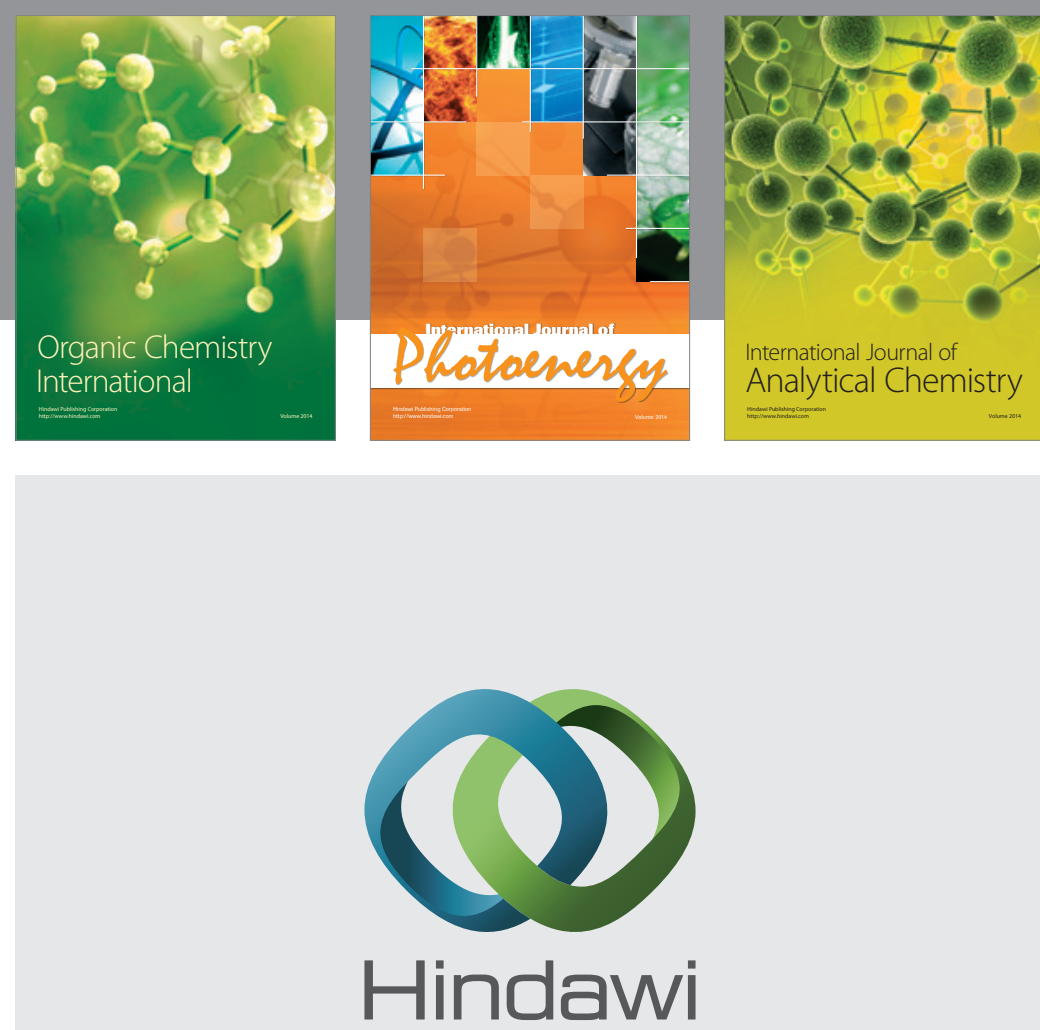

Submit your manuscripts at

http://www.hindawi.com
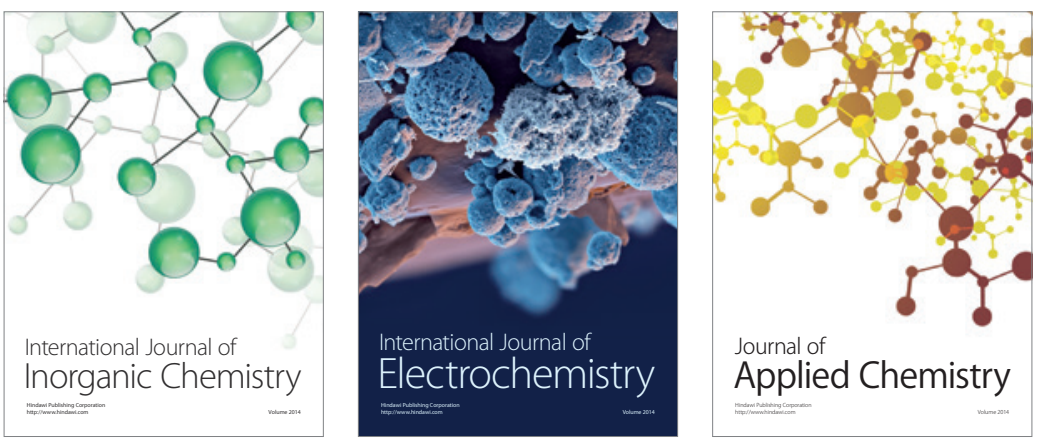

Journal of

Applied Chemistry
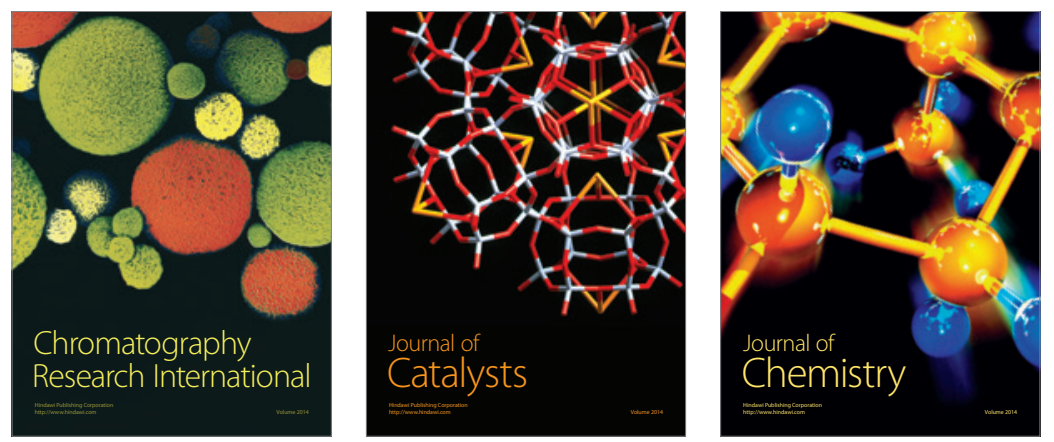
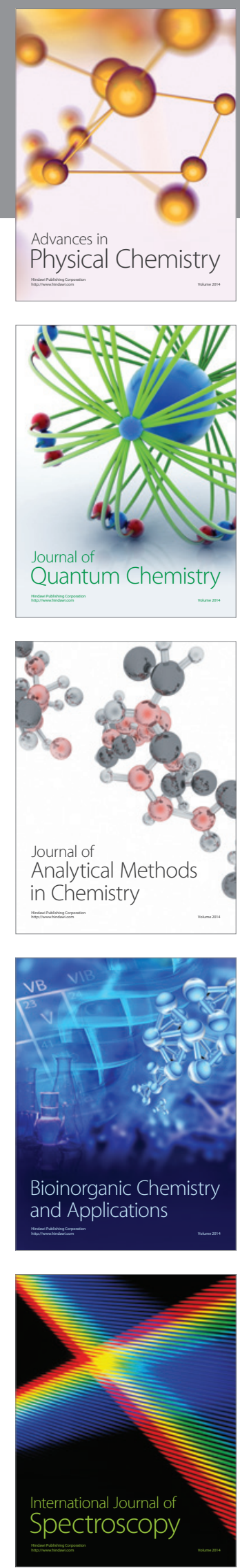